\title{
PREVALENCIA DE ORGASMO EN MUJERES UNIVERSITARIAS DE BUCARAMANGA (COLOMBIA), 2013
}

\section{Prevalence of orgasm in female university students in Bucaramanga (Colombia), 2013}

Rocío Guarín-Serrano, MD, Esp, $M_{S c^{1}}$; Laura Cadena-Afanador, MD, MSP'; Ana María Mujica-Rodríguez, MD, MSc ${ }^{3}$; Miguel Enrique Ochoa-Vera, MD, $\mathrm{MSc}^{4}$; Bernardo Useche-Aldana, $\mathrm{PhD}^{5}$

Recibido: febrero 20/14 - Aceptado: diciembre 15/14

\section{RESUMEN}

Objetivo: establecer la prevalencia de vida de orgasmo femenino y de dificultades orgásmicas en universitarias del área metropolitana de Bucaramanga (Colombia).

Materiales y métodos: estudio de corte transversal. Se administró un cuestionario de autorreporte a una muestra de mujeres universitarias de Bucaramanga. Además de la prevalencia de orgasmo, se midieron variables sociodemográficas como edad, orientación sexual, religiosidad y estado civil; y variables relacionadas con la conducta y la estimulación sexual. Se realizó un análisis descriptivo y un análisis exploratorio mediante análisis bivariado y multivariado para medir la asociación de variables de interés con el orgasmo.

1 Docente de Ginecología y Obstetricia, Universidad Autónoma de Bucaramanga (Colombia).rguarin3@unab.edu.co

2 Coordinadora Académica, Programa de Medicina, Universidad Autónoma de Bucaramanga (Colombia).

3 Estudiante Doctorado Universidade Federal de Santa Catarina, Florianapolis (Brasil).

4 Docente Epidemiología, Universidad Autónoma de Bucaramanga (Colombia).

5 Director del Centro de Investigaciones Biomédicas, Universidad Autónoma de Bucaramanga (Colombia).
Resultados: se encuestaron 1.038 mujeres, con una edad mediana de 20 años. La prevalencia de vida de orgasmo fue del 76\% (IC 95\%: 73,5-78,7) y la prevalencia de dificultades para alcanzar el orgasmo fue del 24\% (IC 95\%: 21,3-26,5). De las mujeres que no habían tenido orgasmo o tenían dificultades para alcanzarlo el 6,2\% habían consultado al ginecólogo por esta razón. En el análisis multivariado se encontró asociación entre el orgasmo y la edad $(\mathrm{OR}=1,19$; IC $95 \%$ : 1,08-1,31), la masturbación (OR $=2,55$; IC $95 \%: 1,34-4,84)$ y las relaciones sexuales con una pareja (OR = 4,2; IC 95\%: 2,28-7,72).

Conclusión: una de cada 3 mujeres universitarias no han tenido experiencia orgásmica y tres de cada 10 mujeres con experiencia orgásmica reportan dificultad para alcanzarlo. Es importante indagar sobre estos problemas sexuales en la consulta de ginecología.

Palabras clave: orgasmo, salud de la mujer, estudiantes universitarias, salud sexual.

\section{ABSTRACT}

Objective: To determine the lifetime prevalence of female orgasm and difficulty reaching orgasm among female university students in the Bucaramanga metropolitan area, Colombia. 
Materials and methods: Cross-sectional study. A self-reporting questionnaire was given to college women in Bucaramanga. Besides the prevalence of orgasm, other social and demographic variables were measured, including age, sexual orientation, religiousness and marital status. Bivariate and multivariate descriptive and exploratory analyses were performed in order to measure the association of the variables of interest with orgasm.

Results: Overall, 1,038 women with a mean age of 20 years were surveyed. Lifetime prevalence of orgasm was $76 \%$ (95\% CI: 73.5 to 78.7 ) and the prevalence of difficulty reaching orgasm was 24\% (95\% CI: 21.3 to 26.5). Among the women who had not experienced orgasm or had difficulties reaching it, 6.2\% had visited a gynaecologist for that reason. The multivariate analysis showed an association between orgasm and age (OR $=1.19$; 95\% CI: 1.08 to 1.31$)$, masturbation (OR $=2.55 ; 95 \% \mathrm{CI}: 1.34$ to 4.84$)$ and intercourse with a partner $(\mathrm{OR}=4.2$; CI 95\%: 2.28 to 7.72 ).

Conclusion: One out of 3 college women have not experienced orgasm and 3 out of every 10 women with orgasmic experience report difficulty reaching it. It is important to ask about these sexual problems during the gynaecological consultation.

Key words: orgasm, women's health, college students, sexual health.

\section{INTRODUCCIÓN}

El orgasmo se define como un clímax de intenso placer sexual que es percibido de manera subjetiva a nivel neurológico central, y se acompaña de manifestaciones periféricas variables, principalmente eventos vasogénicos y miotónicos (1). El orgasmo ocurre ante la tensión sexual creciente producto de la estimulación somato y psicosexual que caracteriza las distintas conductas sexuales (2).

Los fenómenos somato-fisiológicos asociados con la experiencia orgásmica femenina se han estudiado de manera sistemática desde hace cincuenta años (3-6). Se ha descrito que existen factores interpersonales, intrapersonales y socioculturales que influencian la percepción subjetiva del orgasmo en las mujeres $(7,8)$, también se ha descrito que la disfunción orgásmica afecta la salud y calidad de vida de las mujeres $(9,10)$. Los problemas para alcanzar el orgasmo se encuentran clasificados en el Diagnostic and Statistical Manual of Mental Disorders, Fifth Edition (DSM-5) como una disfunción sexual que requiere tratamiento (11), y se han propuesto estrategias de manejo psicológico (12) y ginecológico de las disfunciones orgásmicas femeninas (13).

La frecuencia de disfunción orgásmica varía en cifras que oscilan entre un $3 \%$ y un $33 \%$; por ejemplo, en Estados Unidos, es la segunda disfunción sexual más frecuente, con una prevalencia del $24 \%$ (14). En Chile se ha informado que el $33 \%$ de las mujeres mapuche nunca han tenido un orgasmo (15), que el $33 \%$ de las mujeres nunca han tenido un orgasmo (15). En Colombia, Acuña et al. encontraron un 3,4\% de mujeres con incapacidad para alcanzar el orgasmo y un 15,3\% con dificultades frecuentes para alcanzarlo (16). En un estudio reciente en Medellín se identificó una prevalencia de dificultades del orgasmo de $3 \%$ en estudiantes universitarias (17).

La literatura respecto a la frecuencia de disfunción orgásmica en mujeres universitarias es escasa. Un estudio en una escuela de medicina de Estados Unidos encontró que el $63 \%$ de las estudiantes mujeres se encontraban en alto riesgo de disfunción sexual (18). En Colombia se encuentran algunos estudios que describen las características y dificultades de la fase orgásmica en mujeres universitarias $(17,19)$; específicamente en la ciudad de Bucaramanga, ubicada en una región caracterizada por una tradición de conservadurismo sexual (20), no se ha abordado esta problemática. Por tanto, el objetivo de la investigación fue establecer la prevalencia de orgasmo en universitarias del área metropolitana de Bucaramanga (AMB), y hacer un análisis exploratorio de las dificultades que algunas reportan para alcanzar el orgasmo. 


\section{MATERIALES Y MÉTODOS}

Estudio de corte transversal descriptivo. Los criterios de inclusión fueron: mujeres matriculadas en algún programa de las instituciones de educación superior (IES) ubicadas en Bucaramanga, tener 18 años o más y aceptar voluntariamente el consentimiento verbal para participar. No hubo criterios de exclusión. El universo comprendió a las 31.725 mujeres matriculadas en las 13 IES registradas en el Ministerio de Educación Nacional (9). Se calculó una muestra de 576 mujeres para una prevalencia esperada de $20 \%$ de dificultades en el orgasmo, teniendo en cuenta un nivel de significancia del $5 \%$ y un poder del $80 \%$. Se realizó un muestreo estratificado simple por el tamaño de las IES, las cuales se dividieron en dos grupos, según tuvieran más o menos de 4.000 estudiantes.

Procedimiento. Se solicitó autorización para la recolección de los datos en las 13 IES del AMB, pero solo 7 de ellas dieron su aprobación. Se utilizó como instrumento una encuesta de autorreporte diseñada por los autores, la cual tuvo validación facial y de contenido por tres expertos en el área y por un grupo de estudiantes universitarias. Las variables independientes fueron: edad, estrato socioeconómico, religión, práctica religiosa, ocupación, estimulación genital con y sin pareja y orientación sexual.

La información se digitó en una base de datos en el programa de Epi Info 2012. Los resultados fueron analizados con STATA 11.2, según el tipo de variable, de forma descriptiva con medias y desviación estándar para variables continuas y proporciones e intervalo de confianza del $95 \%$ para variables cualitativas. Se determinó prevalencia de vida. Se comparó el grupo de mujeres con orgasmo con el grupo con problemas orgásmicos mediante la prueba $\chi^{2}$ y prueba exacta de Fischer para las variables cualitativas; y pruebas no paramétrica de Mann Whitney U test para las variables cuantitativas. Se calcularon medidas de asociación (odds ratio) crudas y ajustadas en un modelo de regresión logística con sus respectivos intervalos de confianza al $95 \%$ (IC $95 \%$ ). Se tomó como variable dependiente haber experimentado orgasmo.
Se consideraron significativos los resultados con valores de $\mathrm{p}<0,05$.

Aspectos éticos. El estudio contó con la aprobación del Comité de Ética de la Universidad Autónoma de Bucaramanga. Se solicitó consentimiento verbal para participar en la encuesta, la cual se administró de forma anónima con el fin de garantizar la confidencialidad de las participantes.

\section{RESULTADOS}

Se encuestaron 1.038 mujeres mayores de edad estudiantes de las 7 IES que aceptaron participar. La tasa de preguntas sin contestar en la encuesta fue del $2 \%$. Las participantes, en su mayoría, residían en el área metropolitana (72,45\%), pertenecían a los estratos socioeconómicos 1 al 3 (57,93\%), eran católicas (66,6\%), solteras con pareja (46,9\%), estudiantes que no trabajaban $(66,1 \%)$ y pertenecientes a universidades privadas (62,91\%). La edad de las participantes estaba comprendida entre los 18 y 49 años, con una distribución asimétrica, con una mediana de 20 años y un recorrido intercuartil (RIC) de 4 años. Las principales características sociodemográficas de las participantes se observan en la tabla 1.

La prevalencia de vida de orgasmo fue de 76,0\% (790 casos; IC 95\%: 73,5-78,7). El $55 \%$ (IC $95 \%$ : $52,1-58,1)$ de las participantes (572 casos) manifestó haber tenido orgasmos sin dificultad; el 21\% (IC $95 \%: 18,5-23,5)$ reportó haber tenido orgasmos pero con dificultad (218 casos), y el 24\% restante (248 casos; IC 95 \%: de 21,3-26,5), no tenía experiencia orgásmica al momento de la encuesta.

El rango de edad del primer orgasmo fue entre los 5 a 38 años con una mediana de 18 años (RIC: 3 años). Para las 790 mujeres que habían experimentado orgasmos, la frecuencia orgásmica fue la siguiente: 28 mujeres (3,5\%) tuvieron orgasmos con una frecuencia de una a varias veces al día; 222 mujeres $(28,1 \%)$ una a varias veces a la semana; 366 mujeres $(46,3 \%)$ una a varias veces al mes, y 171 mujeres $(21,6 \%)$ solo lo tuvieron una o varias veces al año. De las 466 mujeres sin orgasmo o con 


\begin{tabular}{|c|c|c|c|}
\hline \multicolumn{2}{|r|}{ Variable } & \multirow{2}{*}{$\begin{array}{c}\text { Frecuencia } \\
831\end{array}$} & \multirow{2}{*}{$\begin{array}{c}\text { Porcentaje } \\
80,06\end{array}$} \\
\hline \multirow{5}{*}{ Edad (años) } & $18-23$ & & \\
\hline & $24-29$ & 120 & 11,56 \\
\hline & $30-35$ & 55 & 5,30 \\
\hline & $36-41$ & 21 & 2,03 \\
\hline & $42-49$ & 11 & 1,06 \\
\hline \multirow{3}{*}{ Estrato socioeconómico } & $1-2$ & 236 & 22,80 \\
\hline & $3-4$ & 703 & 67,92 \\
\hline & $5-6$ & 96 & 9,27 \\
\hline \multirow{5}{*}{ Estado civil } & Soltera con pareja & 487 & 46,9 \\
\hline & Soltera sin pareja & 413 & 39,75 \\
\hline & Casada & 74 & 7,12 \\
\hline & Unión libre & 53 & 5,10 \\
\hline & Separada/divorciada & 12 & 1,15 \\
\hline \multirow{4}{*}{ Orientación sexual } & Heterosexual & 963 & 92,69 \\
\hline & Bisexual & 28 & 2,69 \\
\hline & No definida & 22 & 2,12 \\
\hline & Homosexual & 21 & 2,02 \\
\hline \multirow{4}{*}{ Nivel de estudios } & Técnico o tecnológico & 301 & 29,00 \\
\hline & Universidad incompleta & 701 & 67,53 \\
\hline & Universidad completa & 21 & 2,02 \\
\hline & Posgrado & 15 & 1,45 \\
\hline \multirow{3}{*}{ Ocupación } & Solo estudiante & 684 & 66,1 \\
\hline & Estudia y trabaja (empleada) & 248 & 23,96 \\
\hline & Estudia y trabaja (independiente) & 103 & 9,95 \\
\hline \multirow{4}{*}{ Procedencia } & Área Metropolitana (AMB) & 752 & 72,38 \\
\hline & Santander, fuera del AMB & 96 & 9,24 \\
\hline & Otro departamento & 178 & 17,13 \\
\hline & Sin dato & 13 & 1,25 \\
\hline \multirow{3}{*}{ Pertenencia a religión } & Católica & 692 & 66,60 \\
\hline & Otras & 163 & 15,69 \\
\hline & Ninguna & 184 & 17,71 \\
\hline \multirow{3}{*}{ Consideración de práctica } & Nada o poco practicante & 512 & 49,28 \\
\hline & Moderadamente practicante & 444 & 42,73 \\
\hline & Muy practicante & 81 & 7,80 \\
\hline
\end{tabular}

dificultades para alcanzarlo, 142 (30,4\%) hicieron algún tipo de consulta, 36 mujeres (7,7\%) pidieron consejo a un amigo o amiga; 34 (7,3\%) hicieron consultas por Internet; 29 mujeres $(6,2 \%)$ consul- taron al ginecólogo; 21 (4,5\%) al médico general y las 22 restantes $(4,7 \%)$ al psicólogo, psiquiatra o terapeuta sexual. Se encontró que 98 mujeres de la muestra $(39,5 \%$ de las mujeres sin experien- 
cia orgásmica y 9,4\% del total de participantes) reportaron no haber tenido contacto con algún estímulo somato o psicosexual que pudiera llevar a la excitación o al orgasmo. Los estímulos somato o psicosexuales por los cuales se indagó en este estudio fueron: actividad masturbatoria y coital; estimulación de genitales por una pareja; exposición a materiales eróticos o pornográficos, y haber experimentado fantasías o sueños eróticos.

De las mujeres que habían experimentado orgasmo, solo tres $(0,4 \%)$ reportaron haber estado expuestas a un único tipo de los estímulos mencionados; mientras que las restantes 787 (99,6\%) mujeres manifestaron haber estado expuestas a una diversidad de estímulos sexuales. Las mujeres con experiencia en múltiples tipos de estimulación sexual tienen un 89,7\% de probabilidad de experimentar un orgasmo; mientras que aquellas que han sido expuestas a un único tipo de estimulación, tienen un 4,8\% de probabilidad de alcanzarlo.

En la tabla 2 se comparan de manera bivariada las características sociodemográficas según la probabilidad de alcanzar o no un orgasmo. Se encontró una asociación significativa $(\mathrm{p}<0,01)$ entre la presencia de orgasmo y la edad, la ocupación, dos o más tipos de estímulo y la práctica religiosa.

En el análisis de regresión logística, la edad y las relaciones sexuales con pareja (RSP) mostraron

\begin{tabular}{|c|c|c|c|c|}
\hline \multicolumn{2}{|c|}{ Variables } & $\begin{array}{l}\text { Sin experiencia } \\
\text { orgásmica } \\
\mathbf{n}=248\end{array}$ & $\begin{array}{l}\text { Con experiencia } \\
\text { orgásmica } \\
\mathbf{n}=790\end{array}$ & $p$ \\
\hline \multicolumn{2}{|l|}{ Edad (mediana)* } & 19 (RIC 18-20) & 21 (RIC 19-24) & $<0,001$ \\
\hline Estrato & $\begin{array}{l}\text { Bajo } \\
\text { Medio } \\
\text { Alto }\end{array}$ & $\begin{array}{c}59(25,0 \%) \\
170(24,2 \%) \\
17(17,7 \%)\end{array}$ & $\begin{array}{l}177(75,0 \%) \\
532(75,7 \%) \\
79(82,3 \%)\end{array}$ & 0,33 \\
\hline Religión & $\begin{array}{l}\text { Ninguna } \\
\text { Católica } \\
\text { Otra }\end{array}$ & $\begin{array}{l}37(20,1 \%) \\
164(23,7 \%) \\
47(29,0 \%)\end{array}$ & $\begin{array}{l}147(79,9 \%) \\
528(76,3 \%) \\
115(70,9 \%)\end{array}$ & 0,16 \\
\hline Práctica religiosa $^{\dagger}$ & $\begin{array}{l}\text { Nada practicante/sin religión } \\
\text { Poco/moderadamente practicante } \\
\text { Muy practicante }\end{array}$ & $\begin{array}{l}37(20,1 \%) \\
177(23 \%) \\
33(40,7 \%)\end{array}$ & $\begin{array}{l}147(79,9 \%) \\
594(77,0 \%) \\
48(59,3 \%)\end{array}$ & 0,001 \\
\hline Ocupación & $\begin{array}{l}\text { Estudia y trabaja } \\
\text { Solo estudia }\end{array}$ & $\begin{array}{c}55(15,7 \%) \\
193(28,2 \%)\end{array}$ & $\begin{array}{l}295(84,3 \%) \\
491(71,8 \%)\end{array}$ & $<0,001$ \\
\hline Orientación sexual $^{\dagger}$ & $\begin{array}{l}\text { Bisexual } \\
\text { Heterosexual } \\
\text { Homosexual } \\
\text { No definida }\end{array}$ & $\begin{array}{c}3(10,7 \%) \\
230(23,9 \%) \\
5(23,8 \%) \\
7(31,8 \%)\end{array}$ & $\begin{array}{c}25(89,3 \%) \\
732(76,1 \%) \\
16(76,2 \%) \\
15(68,2)\end{array}$ & 0,33 \\
\hline $\begin{array}{l}\text { Variedad de estímulos } \\
\text { somato o psicosexuales } †\end{array}$ & $\begin{array}{l}\text { Ningún tipo de estímulo } \\
\text { Un único tipo de estímulo } \\
\text { Dos o más tipos de estímulo }\end{array}$ & $\begin{array}{l}98(39,5 \%) \\
59(23,8 \%) \\
91(36,7 \%)\end{array}$ & $\begin{array}{c}0(0 \%) \\
3(0,4 \%) \\
786(99,4 \%)\end{array}$ & $<0,001$ \\
\hline
\end{tabular}

* Prueba no paramétrica de Mann Whitney U

† Prueba de $\chi^{2}$ 
asociación con la presencia de orgasmo. Adicionalmente, se encontró un gradiente de asociación entre la frecuencia de estimulación del clítoris durante la RSP y la posibilidad de orgasmo. El ser muy practicante de alguna religión, la ocupación y la orientación sexual no mostraron significancia estadística con la presencia de orgasmo en el modelo ajustado (tabla 3).

\section{DISCUSIÓN}

Los hallazgos relevantes de este estudio muestran que $24 \%$ de las mujeres nunca han tenido un orgasmo en su vida y que $21 \%$ de las mujeres que han tenido orgasmos tienen dificultad para alcanzarlo. Si de acuerdo con Alzate (2), el orgasmo es un indicador por excelencia del ejercicio saludable de la función erótica, estos resultados proporcionan evidencia de una vida sexual poco satisfactoria en una población joven con alto nivel educativo.

En Colombia, estudios realizados con mujeres universitarias informaron una prevalencia de incapacidad para alcanzar el orgasmo entre el 3,3 y $11,0 \%(17,21,22)$, menor a la encontrada en la presente investigación. Esta diferencia podría explicarse por la influencia todavía existente de la cultura patriarcal y de la tradición de conservadurismo sexual de la región en que se condujo el estudio (20). Nuestros hallazgos son similares a los informados en la literatura internacional, que reporta una frecuencia de anorgasmia del $24 \%$ en mujeres musulmanas en edad reproductiva en general (23); por otra parte, nuestros datos son inferiores a los

\begin{tabular}{|c|c|c|}
\hline Variable & OR ajustado & IC 95\% OR \\
\hline Edad & 1,19 & 1,08 a 1,31 \\
\hline \multicolumn{3}{|l|}{ Práctica religiosa } \\
\hline Nada practicante/sin religión & Referencia & - \\
\hline Poco/moderadamente practicante & 1,14 & 0,64 a 2,05 \\
\hline Muy practicante & 0,55 & 0,71 a 1,92 \\
\hline Trabaja actualmente & 1,16 & 0,71 a 1,92 \\
\hline \multicolumn{3}{|l|}{ Tipo de actividad sexual previa } \\
\hline No ha tenido relaciones sexuales con una pareja (RSP) & Referencia & - \\
\hline RSP sin estimulación clitorídea & 4,2 & 2,28 a 7,72 \\
\hline RSP con estimulación clitorídea rara vez & 22,7 & 11,6 a 44,6 \\
\hline RSP con estimulación clitorídea algunas veces & 38,6 & 21,4 a 69,6 \\
\hline RSP con estimulación clitorídea siempre o casi siempre & 75,3 & 36,5 a 155,3 \\
\hline \multicolumn{3}{|l|}{ Orientación sexual } \\
\hline Heterosexual & Referencia & - \\
\hline Bisexual & 5,4 & 0,44 a 66,4 \\
\hline Homosexual & 0,48 & 0,12 a 1,79 \\
\hline No definida & 0,57 & 0,15 a 2,07 \\
\hline Uso previo de masturbación & 2,55 & 1,34 a 4,84 \\
\hline
\end{tabular}


reportados en Ghana en mujeres en edad reproductiva (se informa una prevalencia de anorgasmia del $71 \%$ ) (24), y más frecuentes que los reportados en Dinamarca (6\%), aunque ese estudio fue realizado en mujeres de 16 a 95 años (25).

Respecto a los factores de riesgo, la literatura referida a la prevalencia de anorgasmia es escasa y está más orientada a la disfunción sexual en general. Najafabady, Salmani y Abedi informan que a menor educación mayor frecuencia de anorgasmia (23), hallazgo que no es consistente con lo encontrado por nosotros. Ellos también informan que la anorgasmia está asociada a la actitud ante el sexo y a condiciones psicológicas como la ansiedad, el dolor o la fatiga. Respecto a la disfunción sexual en general, Zhang et al., en mujeres en edad reproductiva en China, reportan como factores de riesgo la baja frecuencia de sexo y las actitudes tradicionales acerca del mismo (26). Por otra parte, nuestros hallazgos respecto a la edad, que muestran que a mayor edad mayor frecuencia de orgasmo, discrepan con lo informado por Jaafarpour et al. en mujeres en edad reproductiva en Irán quienes informan que a mayor edad mayor riesgo de disfunción sexual. Por otra parte, estos autores señalan que estar desempleada también incrementa el riesgo de disfunción sexual (27).

Dentro de las limitaciones de este estudio está el no haber tenido acceso sino al $54 \%$ de la población de mujeres universitarias de la ciudad debido a que las IES de orientación confesional no autorizaron la aplicación de las encuestas. Por otra parte, el no haber utilizado una encuesta previamente validada, de las múltiples que se encuentran disponibles (28, 29), podría afectar la estimación de la prevalencia de anorgasmia, lo que dificulta la extrapolación de los resultados. Como fortalezas de esta investigación está el tamaño muestral y el bajo riesgo de sesgo de información. Este potencial sesgo de selección puede tener algún efecto en la distribución de la religiosidad y, por tanto, disminuir el poder estadístico de las pruebas de hipótesis que la involucran.

\section{CONCLUSIÓN}

El orgasmo es un componente integral de la función sexual. La relativa alta prevalencia de dificultades para alcanzarlo en mujeres activas sexualmente es un indicador que merece atención en el campo de la salud sexual. Por la especificidad de su práctica clínica, la/el médica/o ginecoobstetra es el profesional al que corresponde atender los problemas sexuales de las mujeres, por lo cual los resultados de este estudio son relevantes para el ejercicio de su actividad profesional.

\section{AGRADECIMIENTOS}

Los autores agradecen a la Universidad Autónoma de Bucaramanga por la financiación del proyecto. Asimismo, reconocen la participación de los miembros del Semillero de Sexualidad Humana y Salud Sexual en el apoyo a la aplicación de las encuestas.

\section{FINANCIACIÓN}

Este proyecto fue financiado por la Convocatoria bianual de investigación de la Universidad Autónoma de Bucaramanga. Resultados preliminares de este estudio fueron presentados el 22 de septiembre 2013 en el 21st Congress of the World Association for Sexual Health, Porto Alegre (Brasil).

\section{REFERENCIAS}

1. Cavalcanti R, Cavalcanti M. Tratamento Clinica das Inadequações Sexuais. São Paulo: Editora Roca; 2006.

2. Alzate H. Sexualidad Humana. Bogotá: Temis; 1997.

3. Masters WH. The sexual response cycle of the human female: vaginal lubrication. Ann NY Acad Sci. 1959;83:301-7.

4. Alzate $\mathrm{H}$, Useche $\mathrm{B}$, Villegas $\mathrm{M}$. Heart rate change as evidence for vaginally elicited orgasm and orgasm intensity. Annals of Sex Research. 1989;2:345-57.

5. Meston CM, Levin RJ, Sipski ML, Hull EM, Heiman JR. Women's orgasm. Annu Rev Sex Res. 2004;15:173-257.

6. Salonia A, Giraldi A, Chivers ML, Georgiadis JR, Levin R, Maravilla KR, et al. Physiology of women's sexual 
function: basic knowledge and new findings. J Sex Med. 2010;7:2637-60.

7. Basson R. The Female Sexual Response: A Different Model. J Sex Marital Ther. 2000;26:51-65.

8. Lucena B, Abdo C. Personal factors that contribute to or impair women's ability to achieve orgasm. International Journal of Impotence Research. 2014;26:177-81.

9. Elsamra S, Nazmy M, Shin D, Fisch H, Sawczuk I, Fromer D. Female sexual dysfunction in urological patients: findings from a major metropolitan area in the USA. BJU International. 2010;106:524-6.

10. Hisasue SI, Kumamoto Y, Sato, et al. Prevalence of female sexual dysfunction symptoms and its relationship to quality of life: a Japanese female cohort study. Urology. 2005;65:143-8.

11. Diagnostic and statistical manual of mental disorders DSM-5. USA: American Psychiatric Association; 2013.

12. Heiman J, LoPiccolo J. Becoming orgasmic: A sexual and personal growth program for women (revised and expanded edition). New York: Prentice Hall; 1988.

13. Useche B. El examen sexológico en las disfunciones excitatorias y orgásmicas femeninas. Rev Terap Sex Clín. Pesquisa e Aspectos Psicossociais. 2001;1:115-31.

14. Laumann E, Gagnon JH, Michael RT, Michaels S. The social organization of sexuality: sexual practices in the United States. Chicago: University of Chicago Press; 2000.

15. Rivera GP, González IR, Vargas Peña ML. Disfunciones sexuales en la mujer mapuche. Rev Chil Urol. 2004;69:68-72.

16. Acuña A, Ceballos MP, Suárez Beltrán, PA. Estudio sobre algunos aspectos del comportamiento sexual femenino. Urol Colomb. 2008;17:79-90.

17. Quintero MT, Gómez M, Uribe JF. Perfil orgásmico en universitarias de ciencias de la salud. Urol Colomb. 2013;22:18-29.

18. Shindel AW, Ferguson GG, Nelson CJ, Brandes SB. The sexual lives of medical students: a single institution survey. J of Sex Med. 2008;5:796-803.
19. Alzate H, Villegas M. Sexual behavior of unmarried Colombian university students in 1990. J Sex Educ Ther. 1994;20:287-98.

20. Gutiérrez de Pineda V. Familia y cultura en Colombia. Medellín: Universidad de Antioquia; 1994.

21. Zuloaga Posada L, Soto Vélez C, Jaramillo Vélez D. Comportamiento sexual y problemas de salud en adultos jóvenes. Universidad de Antioquia, 1991. Bol Of San Pan. 1995;119:212-22.

22. Ceballos Ospino G, Null L, León Botero E. Autoestima y disfunción sexual en estudiantes universitarias de Programas de Salud de Santa Marta (Colombia). Psicol Caribe. 2008;22:50-62.

23. Najafabady MT, Salmani Z, Abedi P. Prevalence and related factors for anorgasmia among reproductive aged women in Hesarak, Iran. Clinics (São Paulo). 2011;66:83-6.

24. Amidu N, Owiredu WK, Woode E, Addai-Mensah O, Quaye L, Alhassan A, et al. Incidence of sexual dysfunction: a prospective survey in Ghanaian females. Reprod Biol Endocrinol. 2010;8:106.

25. Christensen BS, Grønbaek M, Osler M, Pedersen BV, Graugaard C, Frisch M. Sexual dysfunctions and difficulties in denmark: prevalence and associated sociodemographic factors. Arch Sex Behav. 2011; 40: 121-32.

26. Zhang H, Fan S, Yip PS. Sexual Dysfunction among Reproductive-Aged Chinese Married Women in Hong Kong: Prevalence, Risk Factors, and Associated Consequences. J Sex Med. 2014.

27. Jaafarpour M, Khani A, Khajavikhan J, Suhrabi Z. Female sexual dysfunction: prevalence and risk factors. J Clin Diagn Res. 2013;7:2877-80.

28. Olson DH, Fournier DG, Druckman JM. Counselor's manual for PREPARE/ENRICH (revised edition). Minneapolis, MN: PREPARE/ENRICH, Inc.; 1987.

29. Rust J, Golombok S. The GRISS: a psychometric instrument for the assessment of sexual dysfunction. Arch Sex Behav. 1986;15:157-65. 